\title{
ANALYSE D'UNE ORGANISATION HYBRIDE PAR L'APPROCHE DE THOMAS ET HARDY : LE CAS de LA MiSE EN PLACE D'UNe ACTIVITÉ GÉNÉRATRICE DE REVENUS DANS UNE ONG DE DÉVELOPPEMENT EN RÉPUBLIQUE DÉMOCRATIQUE DU Congo
}

\author{
Christelle SuKadi MANGWA $^{1}$
}

Notre recherche se base sur une approche foucaldienne développée par Thomas et Hardy (2014) afin de proposer une analyse critique et longitudinale des discours, comme moyen d'appréhender les tensions entre logiques institutionnelles ainsi que leurs effets sur les organisations hybrides. Inspirée du cadre théorique sur l'intensification des effets pouvoir des auteurs précités, la méthodologie proposée permet de saisir au niveau micro de l'organisation (Orlikowski \& Yates, 2006), la dynamique discursive et les interactions qui accompagnent les acteurs, ainsi que les enjeux de pouvoir conduisant à la mise en place de stratégies organisationnelles. C'est à travers le cas d'une ONG de développement, comprenant deux logiques institutionnelles sujettes à des frictions, que nous étudions les effets des discours sur l'évolution des tensions.

1 Christelle Sukadi Mangwa est doctorante en Information et Communication de l'Université catholique de Louvain et chef de travaux en Sciences de l'Information et de la Communication à l'Université de Lubumbashi (RDCongo).

Recherches en communication, $\mathrm{n}^{\circ} 47$ - Article publié le 28/03/2019 
Notre époque est caractérisée par une hybridation croissante des organisations (Grossi \& Thomasson, 2015). Selon Besharov $\&$ Smith (2014), les chercheurs en sciences de gestion ont utilisé le terme hybride de différentes manières. Néanmoins, l'usage le plus fréquent de ce terme dans les recherches actuelles considère les organisations hybrides comme celles portant en elles des logiques institutionnelles en conflit. Les organisations hybrides sont confrontées aux enjeux relatifs, aux tensions entre logiques susceptibles d'engendrer des conflits (Château Terrisse, 2012). Bien que traduisant une réalité conflictuelle, les tensions entre les logiques institutionnelles sont de plus en plus appréhendées comme positives (Château Terrisse, 2012 ; Michaud, 2011 ; Trethewey \& Ashcraft, 2004). C'est dans l'objectif de comprendre et d'analyser les tensions comme positives ou du moins comme étant à la base d'un revirement positif au sein de l'organisation, que nous nous basons sur le modèle de l' " intensification of power » de Hardy $\&$ Thomas (2014). Ce modèle, qui constitue notre cadre théorique et méthodologique, est basé sur une approche foucaldienne qui permet de prendre en compte les tensions organisationnelles dans leurs dimensions discursives et les implications de la circulation de ces discours en termes de pouvoir.

Foucault conceptualizes power as circulating through discourse, where power works as a 'productive network, which runs through the whole social body' (Hardy \& Thomas, 2014, p. 321).

L'organisation hybride prise comme cas d'étude est le Mouvement des Jeunes pour la Dignité et le Développement (MJDD), une ONG de développement basée en République Démocratique du Congo (ci-après RDCongo). Nous la considérons comme hybride au regard de différentes caractéristiques que nous évoquerons par la suite.

Les organisations hybrides étant confrontées « aux enjeux relatifs, aux tensions entre logiques susceptibles d'engendrer des conflits » (Château Terrisse, 2012) elles permettent d'observer l'évolution conjointe, la confrontation de différentes logiques institutionnelles et constituent donc un cadre propice à l'analyse 
des tensions qui en découlent. À travers le cas du MJDD, nous étudions les tensions dans une dimension discursive et critique, dévoilant les réalités organisationnelles à travers les discours et pratiques des acteurs au niveau micro de l'organisation (Orlikowski \& Yates, 2006).

Nous établirons tout d'abord le lien entre organisation hybride, logiques et tensions. Ceci pour permettre de considérer les organisations hybrides comme des lieux propices à l'analyse des tensions. Nous aborderons ensuite l'approche foucaldienne de Thomas et Hardy (2014) portant sur une analyse critique et longitudinale du discours, afin de montrer le lien existant entre les jeux de pouvoir entre acteurs et la mise en place de stratégies à travers l'évolution des tensions en organisation. C'est à travers le cas du MJDD (Mouvement des Jeunes pour la Dignité et le Développement) que nous nous proposons d'analyser et de comprendre l'évolution des tensions comme positives.

\section{Organisations hybrides, logiques et tensions}

Thornton \& Ocasio (1999) définissent les logiques institutionnelles comme étant des pratiques matérielles, des suppositions, des valeurs, des croyances, des règles qui sont socialement construites et modelées par l'histoire et qui permettent aux individus de vivre, organiser le temps et l'espace et donner du sens à la réalité sociale (Thornton \& Ocasio, 1999, p. 101).

Les logiques institutionnelles sont également définies comme « des systèmes de règles, outils et valeurs associés à des acteurs institutionnels, qui fournissent des lignes d'action et de compréhension en situation " (Charue-Duboc \& Raulet-Croset, 2014, p. 30). Identifier des logiques institutionnelles implique donc de « préciser l'ensemble de croyances, valeurs, outils issus des institutions et qui structurent les actions individuelles et organisationnelles des parties prenantes dans un champs organisationnel » (Charue-Duboc \& Raulet-Croset, 2014, p. 31).

Il existe une relation constitutive entre les logiques et les actions, les unes pouvant renforcer ou déforcer les autres (Thornton \& Ocasio, 1999). 
Lors de la création du MJDD une première logique dominait et amenait les différents acteurs à agir en fonction des objectifs de départ. Il s'agit de la logique sociale. Lorsque dans son évolution, une seconde logique (plus marchande) voit le jour au sein de l'ONG, les comportements, les outils, les actions ont été modifiés, adaptés et ont contribué à renforcer la nouvelle logique. Les acteurs dans une organisation sont en effet des transporteurs, ils représentent et donnent voix aux logiques institutionnelles (Château Terrisse, 2012). Et « même si les logiques influencent la cognition et les actions, les acteurs peuvent à leur tour influencer la manière dont les logiques sont instanciées au sein des organisations » (Besharov \& Smith, 2014, p. 366).

S'agissant des tensions, elles renvoient quant à elles, à des définitions diverses. En effet, la tension est considérée comme positive ou négative en fonction des circonstances, des organisations, ou tout simplement de leurs effets sur l'organisation.

Pour comprendre la notion de tension, nous nous sommes principalement appuyée sur les recherches de Guedri et al. (2014). Ils ont procédé à l'analyse de neuf articles scientifiques basés sur les tensions en contexte organisationnel. Afin de les expliquer, ils reviennent sur les notions de contradiction et de paradoxe. À travers leur revue de littérature, ils présentent la contradiction comme un phénomène au sein duquel des dynamiques et des principes opposés coexistent, alors que chacun d'entre eux a un effet opposé . Deux propositions peuvent être qualifiées de paradoxales si prises isolément chacune d'entre elles est incontestable, alors que considérées simultanément elles apparaissent incohérentes et incompatibles (Guedri et al., 2014, p. 14). Selon les auteurs, les tensions, les paradoxes et les contradictions sont constitutifs de la vie des organisations. Les contradictions donnent parfois lieu à des paradoxes, mais donnent toujours lieu à des tensions (Michaud, 2011).

« Longtemps jugées problématiques, les tensions organisationnelles semblent désormais considérées comme inévitables, indigènes au fonctionnement des organisations dans un environnement post- industriel. Elles sont « normales » et n'ont pas à être 
résolues » (Michaud, 2011, p. 49). Cette conception des tensions est notamment partagée par Trethewey \& Ashcraft (2004).

... we cast paradox, contradiction, and irony-in a word, irrationality- as a rather normal condition of organizational life, not as an anomalous problem to be removed or solved (Trethewey \& Ashcraft, 2004, p. 81).

Les organisations hybrides, au regard de ce qui précède, regorgent de logiques institutionnelles différentes qui sont portées à travers les actions des individus. Ceci nous mène à mettre en avant l'approche critique choisie dans le cadre de cette étude. Cette dernière nous permet de saisir à travers la dynamique des discours, les enjeux de pouvoir (Heracleous \& Barrett, 2001 ; Orlikowski $\&$ Yates, 2006) liés aux tensions. D'où la proposition méthodologique et conceptuelle exposée dans le cadre de notre recherche.

Comme nous l'avons souligné, les logiques institutionnelles en conflit dans les organisations hybrides sont portées par les acteurs de l'organisation (Besharov \& Smith, 2014 ; Michaud, 2011). C'est à travers les interactions des acteurs, à travers les discours produits et leurs effets, que nous étudions les tensions produites au sein de l'organisation hybride et dans notre cas, au sein du MJDD.

Nous décidons de partir sur une approche critique afin de mieux saisir et analyser les tensions dans toute leur évolution en tenant compte des différents enjeux relationnels (Besharov \& Smith, 2014) et de pouvoir des acteurs (Hardy \& Thomas, 2014).

\section{Analyser les tensions à travers une approche critique}

Luc Boltanski dans De la critique. Précis de sociologie de l'émancipation (2009), aborde la critique en opposant d'une part la réalité et d'autre part le monde. Il définit la réalité comme "ce que chacun se représente comme étant "normal" dans une situation donnée, et qui résulte en fait d'une construction sociale cadrée dans une large mesure par les institutions »; le monde, lui, désigne « tout ce qui arrive », « le flux de la vie qui se manifeste 
sans formatage préalable et échappe à tout projet de connaissance totale » (Martinache, 2010, p. 3). Dans cette perspective, la critique consiste à opposer le monde à la réalité. La critique devient donc le dépassement, le fait de chercher à voir ce qui résulte de la confrontation entre la manière dont la réalité est formatée par les institutions et d'autre part le monde qui met l'acteur devant un fait accompli indépendant de son champ d'action. Aussi, « tandis que la sociologie critique analyse des contraintes et des déterminations sociales s'exerçant sur des "agents", la sociologie de la critique s'intéresse aux capacités d'“acteurs" qui ne se réduisent pas à de simples pantins » (Saint-Martin, 2010, p. 2). La critique permet donc d'introduire la notion d'acteur comme élément clé au sein de l'organisation.

Être du côté de la critique signifie donc alors ; attirer inlassablement l'attention sur le reste, sur ce qui n'a pas été symbolisé, sur l'impossibilité contenue dans toute institution ... Il s'agit notamment des rapports de force entre les acteurs, des stratégies à travers lesquelles ces rapports prennent concrètement effets, les jeux qui permettent à ces rapports de se renforcer ou s'affaiblir (Olivesi, 2004). La critique vient dire "vous avez oublié ça", ou "ça ne fonctionne pas comme vous le prétendez" » (Heller, Huët, \& Vidaillet, 2013, p. 26).

Cette façon de penser est d'autant plus pertinente quand on considère les aspects qui influencent la manière dont les logiques prennent place et s'affrontent au sein des organisations.

L'approche critique nous permet ainsi de considérer les effets de la dynamique discursive et donc les enjeux de pouvoir au sein du MJDD. Le choix de l'analyse critique (Heracleous \& Barrett, 2001) et longitudinale des discours se justifie en ce sens qu'elle permet de décrypter les stratégies mises en place au sein des organisations hybrides qui portent différentes logiques, mais aussi de retracer l'histoire de l'émergence et l'évolution de ces tensions. En effet, les études longitudinales permettent d'évaluer non seulement les effets immédiats des tensions mais aussi les effets plus diffus dans le temps (Michaud, 2011). 
C'est donc cette approche critique que nous appliquons à l'étude du cas qu'est les MJDD : une ONG évoluant dans un contexte difficile (Pirotte \& Poncelet, 2002) pour les structures sociales et dans le cadre de laquelle de nouvelles activités ont été développées, introduisant ainsi une nouvelle logique qui entre en tension avec la logique initiale. Suite à la tension entre les deux logiques institutionnelles, une logique sociale et une logique financière, des jeux de pouvoir, des rapports de force se manifestent et renforcent leurs effets à travers des stratégies. De ce qui précède, " on verra intervenir dans les recherches critiques des notions telles que celles de domination, de pouvoir, de rapport de force... » (George \& Carbasse, 2013, p. 183).

Dans une structure comme le MJDD où les acteurs proviennent de plusieurs horizons sociaux, économiques, culturels et avec des intérêts divers, il est important de s'intéresser à ce qui a été « oublié », à « ce qui n'a pas été pris en compte » dans l'analyse communicationnelle des pratiques discursives (Koller, 2009) et dans les tensions. Le contexte de l'ONG donne une place centrale aux enjeux de pouvoir.

Ainsi, l'analyse critique des discours permet de ne pas se limiter à considérer que la logique dominante au sein du MJDD aura été adoptée par « tous ». Au contraire, cette approche nous donne un éclairage qui permet, à travers les effets de discours, de voir comment des jeux de pouvoir se mettent en place et confortent ou déforcent telle ou telle logique institutionnelle, jusqu'à amener l'organisation à adopter de nouvelles stratégies.

\section{L'approche foucaldienne de Hardy et Thomas sur l'intensifi- cation du pouvoir}

Nous avons choisi de nous baser sur la théorie de l'intensification des effets de pouvoir (intensification of power) développée par (Hardy \& Thomas, 2014). Dans leur article, ils procèdent à une analyse critique et longitudinale des discours. Pour ce faire, ils se reposent sur une série de concepts clés des travaux de Michel Foucault que sont : le discours, le réseau de pouvoir et la résistance. 
Foucault ne voit pas le discours comme uniquement composé d'éléments linguistiques : en effet, « Michel Foucault déclare s'intéresser au discours comme "pratique discursive", comme système qui obéit à des règles de formation, de fonctionnement, de coexistence... Pratique qu'il s'efforce de décrire dans "sa consistance et presque sa matérialité” » (Granjon, 2005 , p. 46) :

... le discours ce n'est pas simplement ce qui manifeste (ou cache le désir) ; c'est aussi ce qui est l'objet du désir ; le discours n'est pas simplement ce qui traduit les luttes ou les systèmes de domination, mais ce pour quoi, ce par quoi on lutte, le pouvoir dont on cherche à s'emparer (Foucault, 1971, p. 12)

De plus, le discours circule au sein de l'organisation. Il agit de manière dynamique dans des réseaux de relations. Ils révèlent dans leur pluralité et contradictions, les tensions, conflits, luttes de pouvoir à l'œuvre entre les membres de l'organisation (Mantere \& Vaara, 2008).

D'après Foucault, la conception du pouvoir selon laquelle ce dernier est détenu comme un bien matériel est remise en cause. Comme le précise Bayart (2005, p. 189) en citant L'histoire de la sexualité (1976), « le pouvoir ce n'est pas une institution et ce n'est pas une structure, ce n'est pas une certaine puissance dont certains seraient dotés : c'est le nom qu'on prête à une situation stratégique complexe dans une société donnée ». Foucault conçoit le pouvoir comme " relationnel et réversible » qui s'exerce mais ne se détient pas, il est « action sur des actions », " conduite sur des conduites » (Granjon, 2005, p. 58).

Foucault distingue les « relations de pouvoir », qui sont inévitables, qui autorisent la résistance et la réversibilité et des « états de domination » qui s'instaure quand un groupe réussit à rendre les relations de pouvoir immobiles et fixes, rétrécissant la marge de liberté des sujets (Granjon, 2005, p. 44). L'exercice du pouvoir est donc une relation interactive (Granjon, 2005).

L'exercice du pouvoir, « la gouvernementalité », n'est pas une domination unilatérale, de haut en bas, mais une relation interactive, la « rencontre entre techniques de domination exercées sur 
les autres et les techniques de soi » que l'individu peut mobiliser à son profit. Foucault porte alors toute son attention au «processus de subjectivation $»$, au mode de construction du sujet réactif et inventif (Granjon, 2005, p. 43).

« Tout rapport de pouvoir n'existe que dans un dispositif historique qui articule tant les savoirs, dans lesquels ce pouvoir se code et se décode, que les appareils par lesquels ce pouvoir circule, s'appuie, et touche les corps » (Mazabraud, 2010, p. 131-132).

C'est sur ces arguments de circulation des discours et d'exercice de pouvoir (dans un espace donné et à un moment donné dans un réseau de relations plus ou moins coordonnées) que Hardy et Thomas justifient également leur approche foucaldienne.

Nous analysons donc le pouvoir au sein du MJDD non comme une possession ou un rapport de force figé, de dominants fixes à dominés, mais plutôt comme pouvant permettre justement des résistances et des revirements de situation entre les acteurs. C'est ce qui justifie par rapport au MJDD la posture critique dans laquelle nous nous positionnons.

Le premier concept-clé de l'approche foucaldienne est la résisistance. Au sein de l'organisation, il existe simultanément plusieurs discours. Certains peuvent devenir des résistances au pouvoir, des contre-discours qui vont faire face au discours dominant. Cependant, au lieu de considérer la résistance de manière unidirectionnelle, une réponse adverse avec d'un côté des responsables d'organisation qui veulent implémenter des stratégies et d'un autre côté des employés qui sont contre, Foucault permet de considérer que les résistances et le pouvoir sont itératifs (Hardy \& Thomas, 2014).

Cependant, ces discours de résistance ou contre-discours peuvent, s'ils sont amplifiés, évoluer jusqu'à remplacer le discours principal existant et ainsi perturber le statu quo dans la distribution du pouvoir au sein de l'organisation.

La résistance traduit la tension entre les logiques institutionnelles dans l'organisation hybride. Chacune des logiques, ainsi que les actions qui y sont liées, constituent des discours alternatifs pour l'autre logique. Dans l'évolution du MJDD, face au discours 
principal axé sur le social et présent lors de sa création, évoluent des discours alternatifs qui à leur tour peuvent en s'amplifiant, donner place à l'avènement de la logique financière qui rentre en tension avec la première. C'est notamment cette situation qui est observée par la suite : des résistances mènent à un changement de logique dans le cours de l'évolution de l'ONG.

\section{Le modèle de l'intensification des effets de pouvoir :}

Thomas et Hardy ont constaté dans leur étude (2004) que lorsqu'un discours est porté par des sujets - c'est-à-dire des acteurs qui intériorisent le discours, des personnes qui, de par leurs actions prennent part à ce discours, qui le font vivre à travers leurs agissements et qui le relaient en le mettant en pratique - et des objets - qui sont du matériel, des outils concrets de la mise en œuvre du discours et qui renforcent la présence de ce dernier - il s'intensifie et a tendance à devenir dominant sur les autres discours qui vivent simultanément au sein de l'organisation. L'intensification du pouvoir passe donc par des pratiques qui sont à la fois matérielles et discursives (2004, p. 342), c'est-à-dire la présence des sujets qui interrogent leurs actions (subjectivation) et des objets (objectivation) qui renforcent le discours. Vihalem (2011) précise que le terme « subjectivation » doit surtout être pris dans le sens d'un « rapport à l'ensemble des problèmes posés conjointement par la formation des savoirs et par l'exercice du pouvoir » (Vihalem, 2011, p. 91). Dans cette configuration, le sujet ne cesse de se présenter comme objet d'un savoir et d'un pouvoir. Les subjectivations, en fonction de leur degré et de leur nature « se déploient dans une pensée ou dans un discours et arrivent à modifier la façon dont un objet, un corps est perçu, pensé ou traité » (Vihalem, 2011, p. 92). Le sujet n'est pas «naturel » il est modelé à chaque époque par le dispositif et le discours du moment, il n'existerait pas de sujet humain sans une subjectivation (Veyne, 2008, p. 173). 


\section{À ce propos,}

si on définit la « subjectivation » comme processus par lequel se produit la constitution d'un sujet et de sa soi-disant subjectivité, on peut prétendre que les subjectivations ne se font pas uniquement dans le sens où les sujets se modifient ou sont modifiés selon le calcul du hasard, elles se font également dans le sens où c'est le pouvoir qui doit changer son exercice, cibler ses méthodes et son savoir afin de mieux s'adapter au nombre croissant des situations potentielles d'intervention, à chacun des sujets en question ainsi qu'à chacun des domaines d'application (Vihalem, 2011, p. 97).

Le sujet est constitué, mais le pouvoir peut également apporter son influence en s'adaptant aux configurations auxquelles il fait face. C'est cette subjectivation qui a fait l'objet d'une récupération de Thomas et Hardy qui associent dans leur étude la subjectivation au fait que le discours soit intériorisé, adopté, relayé par des acteurs qui contribuent à le renforcer.

Certaines pratiques viendront au fil du temps renforcer le discours initial avec des objets et des sujets ou au contraire, l'affaiblir. Des contre-discours ou des discours alternatifs vont naître avec le temps (de manière volontaire ou involontaire) et venir contrer les discours d'origine. Ils peuvent s'intensifier, se renforcer à travers des objets et des sujets ou au contraire s'affaiblir à leur tour. Ces affrontements vont conduire à la mise en place de stratégies organisationnelles basées sur le discours dominant.

Si l'on vient aux tensions, en contexte d'organisation hybride, ce cadre théorique permet de retracer l'évolution d'un affrontement entre les logiques institutionnelles qui sont apparues au fil du temps au sein de l'organisation hybride. Il s'agit d'une étude cumulative où en définitive, une logique qui aura été matérialisée par des sujets et des objets domine sur les autres logiques présentes au sein de l'organisation et conduit à la mise en place de stratégies organisationnelles.

La méthodologie proposée est propice à analyser les tensions de manière longitudinale dans la vie de l'organisation à travers les interactions des acteurs et les jeux de pouvoir entre eux (Koller, 2009). 
En nous basant sur le cadre théorique exploité, nous considérons que les logiques institutionnelles évoluent ensemble au fil du temps dans l'organisation. L'une est dominante et les autres sont alternatives. Ces dernières peuvent à leur tour devenir dominantes en fonction de leur matérialisation (présence de sujets et d'objets), en fonction des effets de pouvoir intensifiés. Ainsi, une logique institutionnelle qui aura été subjectivée et objectivée aura plus de chance de devenir la logique dominante au sein de l'organisation. L'intensification est basée sur la répétition du discours dans le temps et par divers acteurs (Butler, 1997) et de l'inscription du discours dans des dispositifs pratiques et matériels (Muniesa et Callon, 2009). Une logique institutionnelle peut être déforcée ou renforcée. C'est en se basant sur ce cadre théorique que nous avons développé la méthodologie qui suit.

\section{Étude de cas}

Depuis quelques dizaines d'années, on constate en Afrique en général la prolifération d'initiatives associatives pour palier le fait que les états ne remplissent pas leur rôle d'acteur du développement économique (Pirotte \& Poncelet, 2002).

C'est dans ce contexte qu'a été créé par un groupe de jeunes étudiants, le Mouvement des Jeunes pour la Dignité et le Développement (MJDD). Une organisation non gouvernementale dont le siège est situé à Lubumbashi en République Démocratique du Congo (RDC). Depuis sa création le 6 décembre 2006, elle est gérée par des jeunes lushois bénévoles dont l'âge moyen est de 25 ans. Le terme ONG est à considérer dans ce contexte, comme une organisation sans but lucratif, créée et gérée exclusivement par des bénévoles.

Le MJDD compte une trentaine de membres effectifs (qui participent aux réunions, votent les décisions importantes de l'ONG, cotisent mensuellement et participent à l'organisation des activités) et plus de 500 membres sympathisants dans la ville de Lubumbashi (les membres sympathisants sont ceux qui participent aux différentes activités sur invitation, ils assurent un taux élevé de participation durant les activités). Il est dirigé par un comité 
sélectionné parmi les mêmes jeunes constitué d'un président (qui en est le fondateur), d'un chargé des relations publiques, d'un secrétaire et de son adjoint, d'un trésorier et d'un commissaire aux comptes, d'un responsable des projets. Les membres de ce comité sont élus à travers l'organisation d'élections auxquelles les membres effectifs participent.

Les objectifs du MJDD sont axés sur la promotion et la consolidation de la paix, la promotion et la vulgarisation des droits de l'enfant, l'écologie et la lutte contre le VIH/SIDA. Dans ce cadre, le MJDD organise des formations pour les jeunes Congolais en leadership et entrepreneuriat, des campagnes de sensibilisation, des conférences, des plantations d'arbres à travers la ville, des activités sportives pour véhiculer des messages de paix, des émissions de radio et de télévision sur la vulgarisation des droits des enfants et la lutte contre le VIH/SIDA. À l'origine de son fonctionnement, le MJDD avait pour seule source de financement les cotisations mensuelles des membres qui, pour la plupart, sont des élèves et des étudiants sans revenu.

Au fil du temps, des partenaires techniques et financiers ont accepté de soutenir le MJDD dans l'organisation de ses activités. Il est ici important de noter qu'à Lubumbashi, contrairement aux villes de l'est du pays souvent en proie aux guerres et aux catastrophes humanitaires, les bailleurs de fonds sont peu nombreux (Pirotte \& Poncelet, 2002). C'est donc un défi pour les ONG locales de se rendre crédibles, de se faire connaître positivement et de développer une image positive afin d'augmenter les chances d'avoir des partenaires financiers. Les financements externes étant faibles, le MJDD comme la plupart des ONG de développement, a dû mettre en place des AGR pour assurer son fonctionnement.

La première AGR du MJDD a été mise en place en 2010. Il s'agit d'un service traiteur qui est constitué à la base d'une douzaine de jeunes de l'ONG. Comme pour les AGR suivantes, $10 \%$ des revenus sont versés dans la caisse de l'organisation. En mettant en place cette AGR, le MJDD a ajouté à ses objectifs sociaux (sa logique sociale), des objectifs liés à la " production» d'argent pour l'organisation de ses activités. Notons que la question d'argent au sein des ONG est souvent un sujet de conflits, de 
suspicions, de détournement (Pirotte \& Poncelet, 2002) et même s'il s'agit de bénévolat, la possibilité d'obtenir un peu d'argent peut être envisagée par les membres.

\section{Méthodologie}

Autant que le cadre théorique, la méthodologie s'inspire des travaux de Thomas et Hardy (2014). Ces derniers établissent un cadre méthodologique basé sur l'analyse des éléments discursifs recueillis. Après avoir déterminé un cadre d'étude, la première étape consiste à rassembler les éléments du discours produits durant la période à observer. Le discours étant considéré comme un ensemble de pratiques, des sources de toutes catégories sont considérées. Ensuite vient l'étape de la construction d'une ligne de temps portant des moments-clés de la période analysée. Ces moments-clés que Lambotte \& Meunier (2013) considèrent comme « kairotiques » sont déterminants, décisifs et pleins de sens dans le cours de l'histoire du phénomène analysé. Il s'agit ici de la construction d'une « event history database » (Van de Ven \& Poole, 1990) qui consiste à l'organisation chronologique des données et leur catégorisation. Cette organisation permet d'identifier le discours principal, les contre-discours ainsi que les personnes qui y sont liées. À travers l'identification de ces clusters discursifs, intervient l'analyse des tensions qui ont lieu entre les logiques au fil du temps dans l'organisation. Notre méthodologie va consister en :

- La collecte des données des discours organisationnels;

- La détermination des moments kairotiques construits sur base de l'analyse des données ;

- L'analyse de la naissance et de l'évolution des logiques institutionnelles à travers les discours des acteurs et l'analyse de la tension entre les logiques institutionnelles;

- L'analyse de la tension entre les logiques en conflit et les stratégies mises en place dans l'organisation.

De manière à analyser concrètement les réseaux de pouvoir (multiplicité des rapports de force, les jeux qui permettent à ces 
rapports d'être renforcés ou déforcés à travers des affrontements, ou encore les stratégies qui leur permettent de prendre effet) (Olivesi, 2004), nous établissons une grille qui nous permet de saisir chacun des éléments qui caractérisent les jeux de pouvoir. Cette grille constitue un guide pour l'analyse concrète des réseaux de pouvoir. Elle comporte :

1) le système des différenciations qui permet d'agir sur l'action des autres (différences juridiques, culturelles, linguistiques, économiques),

2) le type d'objectifs poursuivis par ceux qui agissent sur l'action des autres (privilèges, profits, exercice d'une fonction ou d'un métier),

3) les modalités instrumentales (mécanismes de contrôle, menaces, effets de la parole),

4) les formes d'institutionnalisation (structures juridiques, dispositions traditionnelles, phénomènes de mode ou d'habitude) et les degrés de rationalisation.

Après la description détaillée du déroulement de chaque étape durant l'évolution des tensions, nous présentons à chaque étape dans un tableau, les logiques présentes, les verbatims qui y sont liés, ainsi que les relations de pouvoir dévoilées.

\section{Collecte des données}

Nous avons organisé en 2017, 11 interviews de type semidirectif dont 8 auprès de membres effectifs (ayant accepté volontairement) et trois autres auprès du président, du chargé des relations publiques et du chargé des projets de l'ONG. L'interview consistait à obtenir un récit historique de la mise en place des AGR, racontée par chacun des interviewés. Nous avons également consulté 21 procès-verbaux des réunions contenus dans les carnets du secrétariat (nous avons sélectionné ceux qui étaient directement et indirectement liés à la mise en place et aux activités des AGR). De plus, nous avons consulté 42 rapports d'activités de l'ONG ; le statut notarié de l'organisation ; des supports audiovisuels (11 reportages sélectionnés sur des activités diffusées dans 
des radios et télévisions locales) qui nous ont principalement donné une indication sur le fait que le MJDD accorde une attention particulière à être connu de la population, Nous n'en avons pas analysé le contenu qui est celui des rapports d'activités et des procès-verbaux. Ces supports couvrent la période de 2006 depuis la création du MJDD jusqu'en 2017.

À partir des données récoltées, nous avons effectué un recoupement qui nous a permis de déterminer les logiques institutionnelles au sein de l'ONG. Nous avons organisé ces données de manière chronologique avec les moments « kairotiques » que nous dressons sur une ligne du temps. Dans la reconstitution du récit des interviews, des analyses textuelles des rapports et procès-verbaux ainsi que du statut, les moments kairotiques sont ceux que nous avons établis en tant que chercheur comme étant déterminants, décisifs et pleins de sens dans le cours de l'histoire de la mise en place de l'AGR et dans les affrontements entre les logiques institutionnelles.

Nous reprenons, sur base des moments kairotiques, une série de discours en déterminant leurs tenants, leur dynamique, jusqu'à clairement mettre en lumière les enjeux de pouvoir durant la tension. Ceci nous conduira à comprendre les stratégies organisationnelles mises en place dans le contexte de la tension dont la nature positive est alors révélée. Notre dernière étape va donc effectuer le lien entre l'évolution des logiques et les réalisations concrètes au sein de l'organisation.

\section{Résultats}

De l'analyse des documents et des récits d'interviews, nous avons dégagé deux logiques d'action au sein du MJDD : une logique orientée vers les activités menées en faveur du social et une logique qui comprend les activités destinées à la recherche de fonds. C'est la tension observée entre ces deux logiques qui justifie que le MJDD soit une organisation hybride. Comme dans la plupart des cas, lorsque l'aspect financier est raccordé à une organisation à la base orientée vers une logique tout autre, ceci tend à la naissance de tensions (Nativel, 2017 ; Château Terrisse, 
2012). Nous allons, dans cette section, retracer historiquement à travers les discours, l'évolution de la tension entre les logiques. En prenant en compte la subjectivation, l'objectivation ainsi que les contre-discours, nous présenterons une série de verbatims, extraits de documents qui révèlent concrètement la tension.

La première logique est la logique sociale. Il s'agit de celle qui est liée aux objectifs premiers du MJDD, notamment les activités menées en faveur de la population.

La seconde logique est la logique financière. Elle est axée principalement sur la recherche de fonds, notamment ceux engendrés par les AGR au sein du MJDD. Bien que l'aspect financier intervienne non dans un but de lucre, mais plutôt pour soutenir les activités sociales, il a eu tendance à détourner les membres de l'organisation des objectifs premiers qui sont sociaux. De plus, les ONG dans le contexte congolais sont souvent considérées comme des lieux de détournement de fonds (Pirotte \& Poncelet, 2002). Ceci a conduit à des tensions dont les pratiques des membres ont établi des rapports de force oscillant entre le social et la recherche de fonds.

De l'analyse des différentes sources, nous avons relevé historiquement les temps forts (les moments kairotiques tel que définis) vécus par le MJDD dans l'implémentation de l'AGR. Ce sont les moments qui ont été mobilisés par les interviewés dans leurs récits historiques et une forte production de textes (rapports périodiques, termes de références des projets, projets de développement, procès-verbaux) est axée autour de ces périodes :

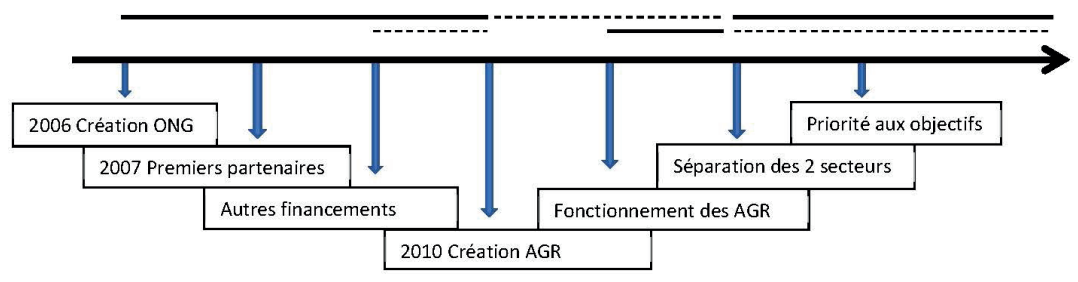

LS :

LF :

Figure 1. Ligne du temps en rapport avec l'implémentation de l'AGR et les deux logiques 
Les étapes importantes de la ligne du temps dans l'évolution des tensions au sein du MJDD sont constituées de la création de l'ONG en 2006 ; l'affirmation, la crédibilisation et les premiers partenaires financiers et techniques en 2007 ; la nécessité d'autres sources de financement; la création d'une AGR en 2010 ; la gestion et le fonctionnement de l'AGR; la séparation des 2 secteurs en 2011 ; la priorisation des activités sociales en 2011. La logique sociale et la logique financière se sont succédé et ont parfois évolué au même moment à des intensités différentes (+ pour la plus forte et - pour la plus faible). Il y a un mouvement de va-et-vient entre ces deux logiques qui se manifeste à travers les activités organisées par l'ONG ainsi que les décisions prises par le comité directeur ou en assemblées générales : les unes portant la dynamique de l'organisation vers les objectifs liés au social et les autres contribuant à réaliser les objectifs fixés sur le plan financier. Les différentes étapes sont porteuses de pratiques, de chevauchement entre logiques et de mises en place stratégiques.

Foucault élargit la compréhension des phénomènes de pouvoir en objectivant ce dernier tel qu'il se déploie dans des procédures, traverse des institutions, structure des champs de pratiques, modèle des comportements, etc. (Olivesi, 2004, p. 46). L'exercice du pouvoir étant un mode d'action de certains sur nous développons l'analyse qui suit, en nous basant sur les étapes importantes de la confrontation entre les logiques au sein du MJDD.

Nous débutons par l'analyse de la première phase qui est la création de l'ONG. À cette étape, l'organisation étant nouvelle et les défis sociaux à relever nombreux, les membres qui joignent le MJDD agissent volontairement en fonction des directives insufflées par le comité directeur. En effet, les textes officiels (le statut et le Règlement d'Ordre Intérieur) guident les nouveaux membres à participer activement à la réalisation des objectifs sociaux.

Les cotisations des membres étaient les principales sources de financement des activités et suffisent à réaliser les premières sensibilisations dans les écoles de la ville. À ce niveau, la logique sociale $(+)$ est présente, entretenue et objectivée principalement par les textes officiels. 
Tableau 1. Verbatims et enjeux de pouvoir liés à la création de l'ONG

\begin{tabular}{|l|l|l|}
\hline $\begin{array}{l}\mathbf{N}^{\circ} \\
\text { d'extrait }\end{array}$ & $\begin{array}{l}\text { Type de } \\
\text { logique }\end{array}$ & Verbatim \\
\hline $\mathbf{1}$ & $\begin{array}{l}\text { LS+ } \\
\text { « ... la jeunesse étant l'avenir, il nous faut être acteurs } \\
\text { du développement de notre pays... Nous sommes un } \\
\text { groupe de jeunes animés par la volonté de contribuer } \\
\text { au développement de notre pays... » (extrait du Statut } \\
\text { de l'ONG notarié en 2007) }\end{array}$ \\
\hline $\begin{array}{l}\text { E n j e u x } \\
\text { de } \\
\text { pouvoir }\end{array}$ & $\begin{array}{l}\text { Les acteurs ayant été présents à la création de l'ONG sont consi- } \\
\text { dérés comme ayant plus d'expérience que ceux qui adhèrent, et } \\
\text { ces derniers agissent en conséquence, renforçant cette différence. } \\
\text { D'office ce qui est écrit, dit, conseillé par le comité directeur est } \\
\text { pris comme une décision indiscutable (le respect de « l'autorité » } \\
\text { étant ancré dans la culture). De plus, faire partie d'une ONG de } \\
\text { jeunes est une chance, un privilège à saisir et à ne pas perdre car } \\
\text { elle donne une bonne image de soi dans la communauté. Ici, les } \\
\text { modalités instrumentales et formes d'institutionnalisation passent } \\
\text { par les effets de la parole, par le Règlement d'Ordre auquel tous } \\
\text { les membres sont soumis. La LS est donc favorisée dans ce } \\
\text { contexte. }\end{array}$ \\
\hline
\end{tabular}

La deuxième phase est celle de l'affirmation, la crédibilisation et l'obtention des premiers partenaires financiers et techniques. Avec la prolifération des associations dans la ville de Lubumbashi, il n'est pas aisé d'acquérir une crédibilité. Or, avoir le crédit de la société civile est un enjeu primordial. Il faut s'imposer, être vu par les bailleurs de fonds, développer une forte notoriété (Pirotte \& Poncelet, 2002).

Le MJDD a pu se démarquer en organisant plusieurs actions auprès des élèves de la ville, tout en prenant le soin d'y inviter différents acteurs de la société civile tel que les organisations religieuses, les groupes socioculturels, les autres ONG de jeunes qui sont de potentiels partenaires, et de diffuser des reportages dans les médias (voir extrait $n^{\circ} 1$ ). L'image de l'organisation s'est forgée aux yeux de la communauté, de la société civile et des partenaires qui décident d'accompagner les activités sur le plan technique et financier (voir extraits $n^{\circ} 2$ et 3 ). 
À ce stade, les financements qui rentrent dans la caisse du MJDD (principalement les cotisations des membres) sont gérés par le comité directeur qui décide de l'attribution des budgets pour les différentes activités sociales à mener. Des rapports financiers sont élaborés et présentés lors des assemblées générales (voir extrait $\left.n^{\circ} 4\right)$.

C'est la logique sociale $(+)$ qui prime étant donné que toutes les actions sont orientées vers la réalisation des objectifs sociaux de l'organisation, même si l'acquisition de partenaires laisse entrevoir un début de logique financière.

Tableau 2. Verbatims et enjeux de pouvoir liés à l'affirmation et la crédibilisation de l'ONG

\begin{tabular}{|l|l|l|}
\hline $\begin{array}{l}\mathbf{N}^{\circ} \\
\text { d'extrait }\end{array}$ & $\begin{array}{l}\text { Type de } \\
\text { logique }\end{array}$ & Verbatim \\
\hline $\mathbf{1}$ & LS & $\begin{array}{l}\text { «Vous savez le MJDD s'est toujours caractérisé par le } \\
\text { travail, nous on travaille et c'est pour cette raison que } \\
\text { les grands (faisant référence aux Nations unies) nous } \\
\text { choisissent comme partenaires. Beaucoup d'autres } \\
\text { jeunes s'étonnent et nous demandent comment on } \\
\text { fait ! (Interview avec le Président, février 2017). }\end{array}$ \\
\hline $\mathbf{3}$ & LS & $\begin{array}{l}\text { La réunion sur le bilan de l'année 2007 permet de } \\
\text { dénombrer une quinzaine de descentes dans des écoles } \\
\text { pour sensibiliser sur la lutte contre le SIDA et les } \\
\text { droits de l'enfant (PV de la réunion du 16 décembre } \\
\text { 2007). }\end{array}$ \\
\hline $\mathbf{3}$ & $\begin{array}{l}\text { Exemple d'une activité : Il a été organisé en date du } \\
\text { 16 juin 2007, à l'école Njanja, la Joumée commémo- } \\
\text { rative de l'Enfant africain ... avec la présence de plu- } \\
\text { sieurs invités de marque dont le représentant du Chef } \\
\text { de Division de la Jeunesse, femme et enfants (extrait } \\
\text { du rapport d'activité de la JEA, 18 juin 2007). }\end{array}$ \\
\hline $\mathbf{4}$ & $\begin{array}{l}\text { La cotisation mensuelle de chaque membre est fixée à } \\
\text { 1\$ US (un dollar américain) ou l'équivalent en Francs } \\
\text { congolais avec conversion au taux du jour (Règlement } \\
\text { d'ordre intérieur annexé au statut, 2007). }\end{array}$ \\
\hline
\end{tabular}




\begin{tabular}{|l|l|}
\hline $\begin{array}{l}\text { Enjeux } \\
\text { de } \\
\text { pouvoir }\end{array}$ & $\begin{array}{l}\text { Sur la scène locale et dans un contexte fort marqué par le tri- } \\
\text { balisme (aussi bien dans la ville que dans le pays) ou encore la } \\
\text { recherche d'argent, plusieurs organisations voient le jour. La plu- } \\
\text { part d'entre elles sont fictives et profitent de financements inter- } \\
\text { nationaux grâce à des réseaux informels d'informations basés } \\
\text { sur des choix tribaux ou des connaissances dans les milieux poli- } \\
\text { tiques. Pour le MJDD, dont la plupart des membres ne sont pas } \\
\text { originaires de la ville de son siège, attirer des partenaires devient } \\
\text { un enjeu de taille. Le MJDD qui décide de s'affirmer, de devenir } \\
\text { visible et d'organiser des activités pour se démarquer des autres } \\
\text { organisations. Ce contexte a des répercussions au sein de l'orga- } \\
\text { nisation où 1'objectif consiste à être crédible et tous les membres } \\
\text { doivent y contribuer : avec une cotisation, avec la surveillance } \\
\text { des listes des cotisations, des listes de présence aux activités. Les } \\
\text { membres en arrivent à se surveiller entre eux car l'enjeu est de } \\
\text { taille. Une fois de plus le Règlement d'Ordre est mis en avant } \\
\text { même si le climat interne suffit à générer un mécanisme interne et } \\
\text { spontané de contrôle entre membres. }\end{array}$ \\
\hline
\end{tabular}

La phase suivante concerne la nécessité pour le MJDD, de posséder d'autres sources de financement. En effet, le MJDD devenant une organisation connue et sollicitée, le nombre d'activités à organiser (conférences, forums) s'est accru. Les cotisations des membres et les supports des partenaires devenaient insuffisants pour couvrir tous les besoins (voir extraits $n^{\circ} 1$ ). Deux camps se créent au sein du MJDD avec d'un côté ceux qui estiment que l'organisation n'a pas besoin de plus d'argent pour fonctionner et de l'autre ceux qui préconisent la mise en place d'une AGR. Les uns en démontrant que les ressources dont dispose le MJDD sont amplement suffisantes pour les objectifs assignés à l'ONG et les autres en émettant des points de vue contraires mettant en avant la nécessité de rechercher des financements. Un rapport de force s'établit entre les deux parties. Ces deux camps sont ici composés l'un et l'autre aussi bien de certains dirigeants que des membres de l'organisation (voir extraits $n^{\circ} 2$ et 3 ).

Les PV montrent que durant cette période la thématique de l'argent revient fréquemment. Pour sortir de cette situation tendue les discussions s'orientent vers « l'avenir financier » du MJDD et 
un consensus est trouvé sur la création d'une AGR (voir extraits $\mathrm{n}^{\circ} 4$ et 5 ).

L'idée du service traiteur, par exemple, naît dans une tension entre les deux parties. Pendant que le projet est en gestation des membres qui étaient d'accord avec la mise en place du service traiteur, achètent à leurs frais des tasses, couverts, divers ustensiles de cuisine pour avoir un matériel de départ et ainsi s'assurer de faire partie de l'AGR (voir extrait $n^{\circ} 6$ ).

Ici la logique financière prend forme au sein de l'organisation. En effet, la mobilisation de certains membres qui vont jusqu'à " offrir » à l'organisation des ustensiles de cuisine pour lancer l'AGR va donner une dynamique (objectiver et subjectiver la LF) ne permettant pas de faire marche arrière sur la création de l'AGR (voir extrait n ${ }^{\circ} 6$ ). En réalité, il s'agissait pour les membres de s'assurer de leur implication dans l'activité. Par ces actions, la logique financière a été intensifiée et nous sommes dans une configuration LS + et LF -.

Tableau 3. Verbatims et enjeux de pouvoir liés à la nécessité d'autres sources de financement

\begin{tabular}{|l|l|l|}
\hline $\begin{array}{l}\mathbf{N}^{\circ} \\
\text { d'extrait }\end{array}$ & $\begin{array}{l}\text { Type de } \\
\text { logique }\end{array}$ & Verbatim \\
\hline $\mathbf{1}$ & LS & $\begin{array}{l}\text { De six activités prévues en début d'année dans le plan } \\
\text { de travail annuel, le MJDD en a réalisé le double. Plu- } \\
\text { sieurs activités se sont greffées au cours de l'année, } \\
\text { notamment après que les responsables d'écoles ou des } \\
\text { médias aient demandé de réorganiser la même acti- } \\
\text { vité après l'intérêt exprimé par les bénéficiaires des } \\
\text { séances de sensibilisation (Réunion de clôture de l'an- } \\
\text { née, 13 décembre 2009). }\end{array}$ \\
\hline $\mathbf{2}$ & LF & $\begin{array}{l}\text { "... nos cotisations ne suffisaient plus à organiser les } \\
\text { activités de l'ONG ... nous avions parfois des activités } \\
\text { non planifiées en debut d'année et les partenaires ne } \\
\text { pouvaient plus nous aligner dans leur budget » (Inter- } \\
\text { view du Chargé des relations publiques, février 2017) }\end{array}$ \\
\hline
\end{tabular}




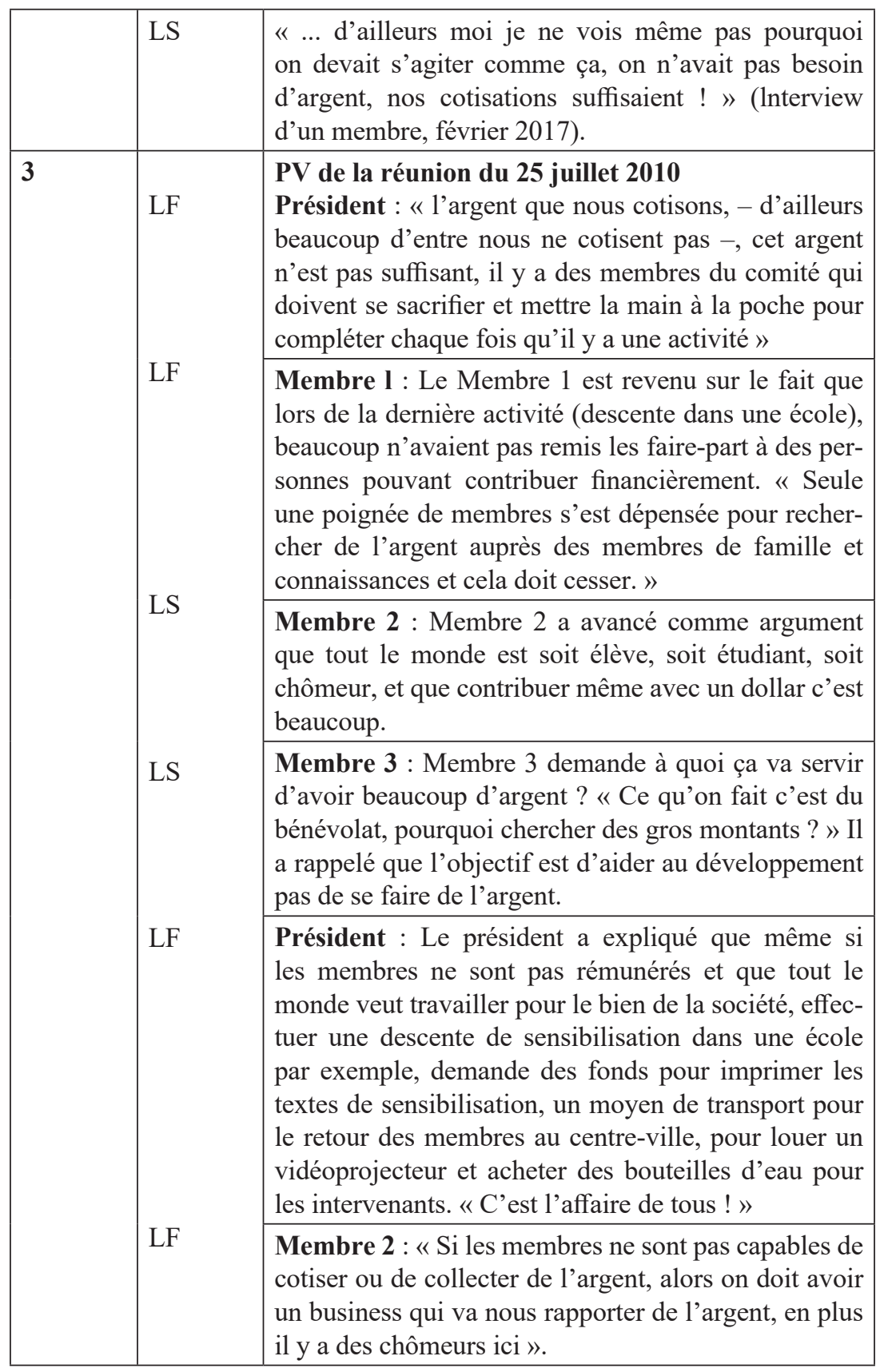




\begin{tabular}{|c|c|c|}
\hline 4 & LF & $\begin{array}{l}\text { Sur quatre réunions successives du comité, du mois } \\
\text { de juillet au mois de septembre, les finances ont été un } \\
\text { des points à l'ordre du jour. }\end{array}$ \\
\hline \multirow[t]{6}{*}{5} & \multirow{2}{*}{$\begin{array}{l}\text { LF } \\
\text { LS }\end{array}$} & $\begin{array}{l}\text { PV de la réunion du } 29 \text { août } 2010 \\
\text { Membre } 1 \text { : «La dernière fois x proposait qu'on ouvre } \\
\text { un business, alors qu'on le fasse !» }\end{array}$ \\
\hline & & $\begin{array}{l}\text { Membre } 2: \text { : } \mathrm{Si} \text { on ouvre un business, les gens } \\
\text { vont nous prendre pour des aventuriers ! On dit par- } \\
\text { tout qu'on est une ONG, tout le monde nous connait } \\
\text { comme étant une ONG!» }\end{array}$ \\
\hline & \multirow[t]{2}{*}{ LF } & $\begin{array}{l}\text { Membre } 3 \text { : «ONG ne veut pas dire qu'on n'a pas le } \\
\text { droit de chercher de l'argent quand même... » }\end{array}$ \\
\hline & & Plusieurs réactions s'enchaînent. \\
\hline & \multirow{2}{*}{$\begin{array}{l}\text { LF } \\
\text { LF }\end{array}$} & $\begin{array}{l}\text { Président : Le président propose de voter et le POUR } \\
\text { l'AGR l'emporte (pas de chiffres). }\end{array}$ \\
\hline & & $\begin{array}{l}\text { Membre } 4: \text { : On peut s'organiser pour commencer à } \\
\text { préparer et servir dans des manifestations. » }\end{array}$ \\
\hline 6 & LF & $\begin{array}{l}\text { «Il y a des jeunes dynamiques qui se sont organisés } \\
\text { pour acheter avec leurs propres moyens, de quoi com- } \\
\text { mencer le service traiteur » (Interview du président, } \\
\text { février 2017). }\end{array}$ \\
\hline $\begin{array}{l}\text { Enjeux } \\
\text { de } \\
\text { pouvoir }\end{array}$ & \multicolumn{2}{|c|}{$\begin{array}{l}\text { Cette étape est chargée en jeux de pouvoir principalement axés } \\
\text { au niveau des différences économiques des acteurs. Il y a un } \\
\text { débat sur le paiement de la cotisation que tous ne peuvent payer, } \\
\text { mais également sur la nécessité de trouver plus de fonds. À tra- } \\
\text { vers les disparités économiques des membres, le discours sur la } \\
\text { LF prend forme. En effet, les membres proviennent de différents } \\
\text { milieux socio-économiques et les discours tenus le révèlent tout } \\
\text { en influençant la mise en place de la LF. Certains refusent de } \\
\text { s'impliquer plus, mais ceux qui ont pour objectif d'apporter plus } \\
\text { d'argent (soit parce qu'ils en ont, soit parce qu'ils en cherchent) } \\
\text { prennent des initiatives en instaurant un dispositif matériel qui } \\
\text { force la mise en place de l'AGR (les ustensiles). Ici, les débats } \\
\text { ainsi que les initiatives de certains en faveur de la LF peuvent } \\
\text { être considérés comme une forme de rationalisation en ce sens } \\
\text { que l'exercice du pouvoir a ici été élaboré, modelé, organisé pour } \\
\text { parvenir à obtenir gain de cause et instaurer un aspect financier } \\
\text { dans les activités de l'ONG. }\end{array}$} \\
\hline
\end{tabular}


La quatrième phase importante de la ligne du temps est la création d'une AGR au sein du MJDD. C'est le moment où est intervenue en priorité la logique financière $(+)$. Il s'agit de la période de mise place du service traiteur (voir extrait $n^{\circ} 1$ ).

L'AGR est mise sur pied et il y a donc matérialisation du discours de mise en place de l'AGR, vu que l'ONG possède déjà quelques ustensiles, de la main-d'œuvre (des membres volontaires). Les pratiques liées aux finances deviennent à ce stade dominantes et renversent celles basées sur la logique sociale.

L'argent devient le centre des discussions et certaines activités du plan d'action ne sont pas réalisées pour privilégier des marchés gagnés à l'improviste au cours de l'année. Suite à cela, des membres craignent que l'argent draine de nouveaux problèmes (voir extrait $\mathrm{n}^{\circ} 2$ ).

Les marchés se succèdent et le MJDD est d'ailleurs souvent contacté pour assurer le service via appel d'offres par les Agences du Système des Nations unies (Journée internationale de la paix, Journée des casque bleus, formation aux élections).

Au sein de l'organisation, la logique financière et la logique sociale évoluent en tension car dans les deux camps, et malgré la création de l'AGR, chacun campe sur ses positions. En effet, la mise en place du service traiteur n'a pas mobilisé toute l'ONG. La logique financière est intensifiée et prend le dessus car elle est renforcée par la décision de sa création (l'AGR), la disponibilité de certains membres à y travailler, ainsi que l'acquisition des premiers marchés. La logique sociale se voit déforcée car des activités sociales qui étaient prévues dans le plan d'action annuel sont annulées pour privilégier les marchés acquis pour le service traiteur. La configuration est LF+ et LS - à ce stade. 
Tableau 4. Verbatims et enjeux de pouvoir liés à la création de l'AGR

\begin{tabular}{|c|c|c|}
\hline $\begin{array}{l}\mathrm{N}^{\circ} \\
\text { d'extrait }\end{array}$ & $\begin{array}{l}\text { Type de } \\
\text { logique }\end{array}$ & Verbatim \\
\hline 1 & $\mathrm{LF}$ & $\begin{array}{l}\text { «... le service traiteur » va permettre de couvrir à une } \\
\text { certaine hauteur les activités que nous organisons » } \\
\text { (PV de la réunion d'annonce de la mise en place de } \\
\text { l'AGR, } 26 \text { septembre 2010). } \\
\text { «... mettre en place cette activité nous a encouragés à } \\
\text { l'esprit de créativité et d'entrepreunariat » (Interview } \\
\text { avec un membre de l'ONG, février 2017). }\end{array}$ \\
\hline 2 & $\mathrm{LF}$ & $\begin{array}{l}\text { «... partout où il y a de l'argent à gérer, surtout ici au } \\
\text { Congo, ça amène toujours à des problèmes ! » (extrait } \\
\text { d'une interview avec un membre, février 2017). } \\
\text { Selon le plan de travail annuel, le MJDD devait orga- } \\
\text { niser } 2 \text { conférences entre septembre et décembre mais } \\
\text { en même temps, il fallait organiser le service trai- } \\
\text { teur pour des clients. Les conférences ont donc été } \\
\text { annulées (PV de la réunion sur le bilan de l'année, } \\
19 \text { décembre 2010). }\end{array}$ \\
\hline $\begin{array}{l}\text { Enjeux } \\
\text { de } \\
\text { pouvoir }\end{array}$ & \multicolumn{2}{|c|}{$\begin{array}{l}\text { À la suite des différentes interactions, l'ONG en arrive à la créa- } \\
\text { tion d'une AGR. Qu'il s'agisse de la décision de création ou } \\
\text { même du choix sur le type de business à mettre en place, ce son } \\
\text { les membres les plus anciens ou encore ceux motivés par des idées } \\
\text { entrepreunariales qui imposent leur. discours. L'accumulation de } \\
\text { profits est un des objectifs poursuivis, et toujours avec un sou- } \\
\text { bassement de disparités économiques, leur pouvoir s'institution- } \\
\text { nalise à travers une redistribution des tâches. Le jeu de pouvoir } \\
\text { s'effectue durant les réunions, à travers des débats et même dans } \\
\text { la recherche de marchés. Le discours sur la LF en sort renforcé } \\
\text { La création de l'AGR est en soi la stratégie à travers laquelle les } \\
\text { rapports de force prennent effet. }\end{array}$} \\
\hline
\end{tabular}

La cinquième étape concerne la gestion et le fonctionnement de l'AGR. Cette période comprend les premiers mois d'activité du service traiteur qui fonctionnait avec les membres de l'ONG chargés d'organiser et de participer aux activités sociales.

Les deux logiques évoluent ensemble une fois de plus et la situation de tension qui existait sur la nécessité ou pas de mettre en place l'AGR s'est apaisée. En effet, le président de l'organisa- 
tion a multiplié les rencontres avec tous les membres, rencontres durant lesquelles les bienfaits de l'AGR étaient mis en avant. Il ne s'agissait pas uniquement de la parole du président mais aussi des résultats concrets sur le terrain car les finances apportées par l'AGR amélioraient également la qualité des activités sociales organisées.

Pour preuve, le MJDD ouvre son premier compte bancaire dont le numéro est désormais repris en en-tête de tous les documents (voir extrait $n^{\circ} 1$ ).

Avec le temps, les réunions mensuelles se transforment en compte rendus financiers. Alors qu'avant la mise en place du service traiteur les rapports financiers étaient présentés par le trésorier durant les assemblées générales (en moyenne 4 pour l'année), ils sont désormais présentés à chaque réunion (voir extrait $n^{\circ} 2$ ).

De plus, vu que seuls certains membres (des volontaires au début et ceux désignés par le comité directeur par la suite) travaillent dans l'AGR (ces derniers bénéficiant d'une " petite " prime à chaque prestation), de la convoitise et des frustrations naissent dans le chef de certains, allant même jusqu'à des soupçons de mauvaise gestion d'argent (voir extrait $n^{\circ} 3$ ).

Des membres ayant adhéré décident de quitter l'ONG parce qu'ils ne s'y retrouvent plus en termes d'objectifs sociaux pour lesquels ils y ont adhéré (voir extrait $n^{\circ} 4$ ).

Bien que la mise en place de l'AGR fut rentable pour le MJDD, la logique financière soutenue a été néfaste pour les objectifs de l'organisation. En effet, la logique sociale (-) se déforçait progressivement pour laisser place à la logique financière $(+)$ renforcée par les effets des pratiques des acteurs au sein de l'organisation. L'AGR supposée rapporter de l'argent pour les activités sociales s'est transformée en une activité quasi autonome qui empiète sur la réalisation des activités sociales et fait diminuer les effectifs. 
Tableau 5. Verbatims et enjeux de pouvoir liés à la gestion et au fonctionnement de l'AGR

\begin{tabular}{|c|c|c|}
\hline $\begin{array}{l}\mathrm{N}^{\circ} \\
\text { d'extrait }^{\prime}\end{array}$ & $\begin{array}{l}\text { Type de } \\
\text { logique }\end{array}$ & Verbatim \\
\hline 1 & LF/LS & $\begin{array}{l}\text { Au départ, les cotisations mensuelles pouvaient être } \\
\text { gardées par le trésorier et le commissaire aux comptes, } \\
\text { mais avec le service traiteur il y avait plus d'argent, il } \\
\text { fallait mieux gérer. C'est pourquoi nous avons ouvert } \\
\text { un compte bancaire signé par } 3 \text { personnes. Nous } \\
\text { avons d'aillleurs communiqué ce numéro à nos parte- } \\
\text { naires et à toutes les personnes qui veulent nous aider } \\
\text { (Interview du chargé de projets, février 2017). }\end{array}$ \\
\hline 2 & $\mathrm{LF}$ & $\begin{array}{l}\text { "À chaque réunion mensuelle, un rapport financier } \\
\text { devait être présenté alors qu'avant c'était moins fré- } \\
\text { quent, seulement pendant les assemblées générales » } \\
\text { (extrait de l'interview du chargé de projets, février } \\
\text { 2017). }\end{array}$ \\
\hline 3 & LF & $\begin{array}{l}\text { Ce sont toujours les mêmes personnes qui étaient } \\
\text { choisies pour le travail (Interview d'un membre, } \\
\text { février 2017). }\end{array}$ \\
\hline 4 & $\mathrm{LF} / \mathrm{LS}$ & $\begin{array}{l}\text { «Moi je connais au moins deux personnes qui ont } \\
\text { quitté l'ONG parce qu'elles ne se retrouvaient plus, ... } \\
\text { à moi ils me l'ont dit, même si devant tout le monde } \\
\text { ils disent ne plus avoir de temps pour ça » (Interview } \\
\text { RP, février 2017). }\end{array}$ \\
\hline $\begin{array}{l}\text { Enjeux } \\
\text { de } \\
\text { pouvoir }\end{array}$ & \multicolumn{2}{|c|}{$\begin{array}{l}\text { L'AGR produit des bénéfices, mais les membres attachés au } \\
\text { social, ou frustrés par cette situation se révoltent contre le choix } \\
\text { de ceux qui travaillent dans l'AGR, ou encore s'indignent du fait } \\
\text { que l'ONG se tourne principalement vers les finances dans ses } \\
\text { activités. Ici les différences dans les rapports de force portent } \\
\text { notamment sur la place des membres dans le fonctionnement de } \\
\text { l'AGR. } \\
\text { Être choisi pour travailler dans le service traiteur est une aubaine } \\
\text { car la plupart des membres sont des étudiants et ont besoin } \\
\text { d'argent (bien que l'ONG soit bénévole). L'exercice du pouvoir } \\
\text { ici s'élabore d'une part à travers la gestion de l'AGR avec ceux } \\
\text { qui imposent leur vision des activités, ceux qui désignent les per- } \\
\text { sonnes pouvant travailler dans l'AGR, ceux qui n'hésitent pas } \\
\text { à faire intervenir leur finances privées; et d'autre part ceux qui } \\
\text { dénoncent ces « injustices » dans le choix des acteurs de l'AGR, }\end{array}$} \\
\hline
\end{tabular}




\begin{tabular}{|l|l|}
\hline $\begin{array}{l}\text { ou encore ceux qui décident de « salir » l'image que le MJDD a } \\
\text { construite en décidant de quitter l'organisatiobn (une structure qui } \\
\text { perd des membres est sujette à des critiques de la part des autres } \\
\text { organisations). C'est ainsi que la LS et la LF se sont affrontées sur } \\
\text { le terrain de l'argent et de l'image de l'ONG. }\end{array}$ \\
\hline
\end{tabular}

Après une dizaine de mois d'activité, intervient la sixième étape qui est celle de la séparation des deux secteurs. Les membres de l'organisation travaillant dans l'AGR ont noté des difficultés de fonctionnement de gestion du temps qui devenait complexe, étant donné que les mêmes personnes étaient chargées de tenir l'AGR, mais aussi d'organiser les activités sociales du MJDD (voir extrait $\left.\mathrm{n}^{\circ} 1\right)$.

Après des séances d'échanges et de réflexions sur les problèmes de gestion de temps et de ressources mobilisées par les deux logiques institutionnelles, une proposition est née des interactions entre les membres et le comité. Il est alors question de séparer les deux secteurs et d'allouer à chacun d'entre eux les moyens de fonctionner de manière autonome tout en étant liés.

Ici la logique sociale et la logique financière évoluent ensemble le temps de la stabilisation même si la logique sociale reprend le dessus progressivement (LS + et LF -).

À partir d'octobre 2011 des dispositions concrètes sont prises : il s'agit notamment de la modification de la structure organisationnelle de l'organisation avec la création de postes de responsables pour la gestion de l'AGR jusque-là dirigée par le comité (voir extrait $\mathrm{n}^{\circ} 2$ ).

Ainsi, va être créée une zone de stabilité entre les deux logiques qui ont en fin de comptes restructuré l'organisation. Les tensions ont été productives car elles ont mené l'ONG à adopter une nouvelle structure plus efficace et plus rentable tout en maintenant un équilibre avec les objectifs sociaux originaux. 
Tableau 6. Verbatims et enjeux de pouvoir liés à la séparation des deux secteurs

\begin{tabular}{|l|l|l|}
\hline $\begin{array}{l}\mathbf{N}^{\circ} \\
\text { d'extrait }\end{array}$ & $\begin{array}{l}\text { Type de } \\
\text { logique }\end{array}$ & Verbatim \\
\hline $\mathbf{1}$ & $\begin{array}{l}\text { LS/LF } \\
\text { « Il y avait de quoi se plaindre, nous étions au four et } \\
\text { au moulin ! (Interview d'un membre, février 2017). } \\
\text { « .. ça devenait difficile pour nous de s'organiser et } \\
\text { d'être efficace aussi bien pour le service traiteur que } \\
\text { pour les activités à mener en dehors du service... » } \\
\text { (Interview d'un membre, février 2017). }\end{array}$ \\
\hline $\mathbf{2}$ & $\begin{array}{l}\text { LS/LF } \\
\text { « Nous avons adapté notre organigramme, ceux qui } \\
\text { travaillent dans l'AGR n'ont plus les mêmes obliga- } \\
\text { tions que les autres membres » (Interview du chargé } \\
\text { de projets, février 2017) }\end{array}$ \\
\hline $\begin{array}{l}\text { Enjeux } \\
\text { pouvoir }\end{array}$ & $\begin{array}{l}\text { Face à l'ampleur de la situation : d'une part des membres épui- } \\
\text { sés par le travail et d'autre part le risque d'être sous le feu des } \\
\text { critiques par baisse du nombre d'activités sociales, un équilibre } \\
\text { est envisagé. L'objectif poursuivi ici est la pérennité de l'orga- } \\
\text { nisation. Les relations de pouvoir ici portent sur la nécessité de } \\
\text { poursuivre les activités de l'organisation. La modalité instrumen- } \\
\text { tale est instaurée par les effets de la parole dans les débats lors des } \\
\text { réunions où les membres se plaignent de l'incapacité à travailler } \\
\text { en faveur des activités de la LF et de la LS en même temps. Les } \\
\text { relations de pouvoir s'institutionnalisent concrètement dans la } \\
\text { modification de l'organigramme organisationnel. Le pouvoir de } \\
\text { ceux portant la LS s'est doté d'une procédure formelle, juridique, } \\
\text { qui a conduit au renforcement des activités portées par la LS. }\end{array}$ \\
\hline
\end{tabular}

La dernière étape dans le déroulement des tensions entre logiques est la priorisation des activités sociales. En effet, en décembre 2011, lors de l'élaboration du Plan de Travail Annuel de 2012, il y a un retour vers les activités sociales $(+)$ en priorité. Selon les propos des membres interviewés, les réunions, les activités et les projets du MJDD se sont tournés à $80 \%$ vers l'atteinte des objectifs de l'organisation. Seul $20 \%$ du temps global d'activité se concentre sur l'évolution de l'AGR fonctionnant désormais comme une entité annexe qui fait rapport à l'ensemble du groupe lors des assemblées générales (voir extrait $\mathrm{n}^{\circ} 1$ ). 
Tableau 7. Verbatims et enjeux de pouvoir liés à la priorité des activités sociales

\begin{tabular}{|l|l|l|}
\hline $\begin{array}{l}\mathbf{N}^{\circ} \\
\text { d'extrait }\end{array}$ & $\begin{array}{l}\text { Type de } \\
\text { logique }\end{array}$ & Verbatim \\
\hline $\mathbf{1}$ & LS & $\begin{array}{l}\text { " Après tout, nous ne devions pas oublier la raison } \\
\text { pour laquelle l'ONG a été créée. L'AGR est là pour } \\
\text { nous aider et nous ne devons pas y mettre beaucoup } \\
\text { d'attention » (Interview d'un membre, février 2017). }\end{array}$ \\
\hline $\begin{array}{l}\text { Enjeux } \\
\text { de } \\
\text { pouvoir }\end{array}$ & $\begin{array}{l}\text { C'est le savoir-faire et aussi les compétences des membres por- } \\
\text { tant la LS qui renforcent le discours social. Les objectifs poursui- } \\
\text { vis par cette catégorie de personnes ainsi que les enjeux autour de } \\
\text { l'établissement de la LS comme prioritaire, portent sur la pérenni- } \\
\text { sation de l'ONG et sur le besoin de maintenir une image positive } \\
\text { face à la population, aux pouvoirs publics ou encore aux bail- } \\
\text { leurs de fond. Leur pouvoir s'exerce à travers des règles établies } \\
\text { en séparant les deux logiques. En insistant sur le fait que l'ONG } \\
\text { est une organisation créée pour le bien social, et par le fait que } \\
\text { les activités organisées se fondent sur cette logique, les membres } \\
\text { renforcent et institutionnalisent leur pouvoir. Les deux logiques } \\
\text { finissent par cohabiter même si la LS est dominante à ce niveau. }\end{array}$ \\
\hline
\end{tabular}

La logique sociale originelle a été bouleversée par une autre logique qui a pris le dessus à un moment donné dans l'évolution de l'ONG. En effet, la logique financière s'est renforcée à travers la présence de sujets et d'objets qui l'ont intensifiée. Néanmoins, la logique initiale a repris le dessus à travers un processus de négociations et d'interactions entre les membres de l'organisation. Les enjeux de pouvoir ont été pour beaucoup dans le déroulement de la tension entre les logiques institutionnelles au sein du MJDD. L'effort d'adaptation à la tension entre les logiques, motivé notamment par la volonté d'assurer la pérennité de l'organisation, a donc été productif. En effet, cette adaptation a permis au MJDD de revoir son fonctionnement et de finalement mettre sur pied une nouvelle structure organisationnelle comprenant à la fois logique sociale et logique financière. 


\section{Conclusion}

Dans cet article, nous avons mobilisé un cadre théorique et méthodologique permettant d'appréhender les tensions dans leur dimension discursive. Les organisations hybrides, par leurs logiques institutionnelles en conflit, sont des lieux propices et intéressants pour analyser les enjeux de pouvoir ainsi que la nature productive des tensions. Pour le cas du MJDD, bien que la logique financière et par là toutes les activités menées en ce sens ne soient pas purement destinées à des fins lucratives, la tension est provenue du fait que les activités sociales ont fini par être négligées. En effet, la tension entre les logiques concerne le fait que la logique financière $\mathrm{a}$, à travers les jeux de pouvoirs, contribué à affaiblir les objectifs premiers de l'organisation qui sont en faveur du social.

Pour analyser cette tension, nous nous sommes basée sur l'approche foucaldienne développée par Thomas et Hardy (2014) en effectuant une analyse critique et longitudinale des discours produits au sein d'une ONG, laquelle a vu se chevaucher dans son évolution deux logiques tantôt en accord, tantôt en conflit.

L'évolution de ces affrontements a donné lieu à une restructuration bénéfique au fonctionnement de l'organisation. Bien que des littératures proposent déjà de considérer les tensions comme positives pour l'organisation, nous y contribuons avec des éléments d'ordre méthodologique basés sur l'analyse critique des discours tel que (Hardy \& Thomas, 2014). Car comme le précise Olivesi (2004), l'analyse foucaldienne du discours permet de saisir les discours dans leur fonctionnalité pratique comme " des blocs tactiques dans le champ des rapports de force » ou comme des lieux d'articulation du pouvoir et du savoir (2004, p. 3). L'analyse critique du discours permet en effet de mettre en lumière, historiquement, les enjeux de pouvoir durant la tension, afin de mieux la cerner au niveau micro de l'organisation. 


\section{Références}

Bayart, J.-F. (2005). Foucault au Congo. Dans Marie-Christine Granjon (Éd.), Penser avec Michel Foucault, Théorie critique et pratiques politiques. Coll. Recherches internationales (pp. 183-222). Paris : Karthala.

Besharov, M. L. \& Smith, W. K. (2014). Multiple Institutional Logics in Organizations: Explaining Their Varied Nature and Implications. Academy of Management Review 39(3), 364-381.

Charue-Duboc, F. \& Raulet-Croset, N. (2014). Confrontation de logiques institutionnelles et dynamique des routines organisationnelles, Competing institutional logics and the evolution of organizational routines. Revue française de gestion 240, 29-44.

Château Terrisse, P. (2012). Le dispositif de gestion des organisations hybrides, régulateur de logiques institutionnelles hétérogènes? Le cas du capital-risque solidaire. Management \& Avenir 54(4), 145.

Deleuze, G. (1986). Foucault. Paris : Les Éditions de Minuit.

Foucault, M. (1971). L'ordre du discours. Paris : Gallimard.

George, E. \& Carbasse, R. (2013). Quelle place pour les travaux d'orientation critique en communication dans les revues scientifiques? Dans Thomas Heller, Romain Huët, Bénédicte Vidaillet (Éds). Communication et organisation : perspectives critiques (pp. 181-192). Villeneuve d'Ascq : Presses universitaires du Septentrion.

Granjon, M.-C. (2005). Introduction. Penser autrement avec Michel Foucault, La « méthode historico-critique », usages théoriques, heuristiques et politiques. Dans Marie-Christine Granjon (Éd.), Penser avec Michel Foucault, Théorie critique et pratiques politiques. Coll. Recherches internationales (pp. 5-71). Paris : Karthala.

Grossi, G. \& Thomasson, A. (2015). Bridging the Accountability Gap in Hybrid Organizations: The Case of Malmö-Copenhagen Port. International Review of Administrative Science 81(3), 604-620.Disponible à : https://doi.org/10.1177/0020852314548151.

Guedri, Z., Hussler, C. \& Loubaresse, É. (2014). Contradictions, paradoxes et tensions en contexte(s). Revue française de gestion 240, 13-28.

Hardy, C. \& Thomas, R. (2014). Strategy, Discourse and Practice: The Intensification of Power: Strategy, Discourse and Practice. Journal of Management Studies 51(2), 320-348.

Heller, T., Huët, R. \& Vidaillet, B. (Éd.). (2013). Introduction. Dans Thomas Heller, Romain Huët, Bénédicte Vidaillet (Éds). Communication et organisation : perspectives critiques (pp. 21-28). Villeneuve d'Ascq : Presses universitaires du Septentrion.

Heracleous, L. \& Barrett, M. (2001). Organizational change as discourse: Communicative actions and deep structures in the context of information technology implementation. Academy of Management Journal 44(4), 755-778.

Koller, V. (2009). Analyser une identité collective en discours : acteurs sociaux et contextes. Semen. Revue de sémio-linguistique des textes et discours (27). Disponible : http://journals.openedition.org/semen/8676.

Lambotte, F. \& Meunier, D. (2013). From bricolage to thickness: making the most of the messiness of research narratives. Qualitative Research in Organizations and Management: An International Journal 8(1), 85-100.

Macmillan, A. (2008). Pouvoir, discipline et communication - Articles du congrès 2008 de la SFSIC. Disponible à : http://www.sfsic.org/congres_2008/spip.php?article55 
Mantere, S. \& Vaara, E. (2008). On the Problem of Participation in Strategy: A Critical Discursive Perspective. Organization Science 19(2), 341-358.

Martinache, I. (2010). Boltanski Luc, De la critique. Précis de sociologie de l'émancipation. Coll. NRF Essais. Paris : Gallimard. Comptes rendus. Sociologie. Disponible à http://journals.openedition.org/sociologie/117

Mazabraud, B. (2010). Foucault, le droit et les dispositifs de pouvoir. Cités 2(42), 127-189.

Michaud, V. (2011). Proposition pour l'étude des tensions dans le mouvement, la sociomatérialité et le paradoxe. Communiquer. Revue de communication sociale et publique 5, 47-74.

Nativel, J.-B. (2017). Manager les tensions paradoxales dans l'économie sociale et solidaire. RECMA 344, 74-88.

Olivesi, S. (2004). Sur les logiques d'appropriation de Michel Foucault par les sciences de la communication, 2004(1), 40-49.

Orlikowski, W. J. \& Yates, J. (2006). ICT and Organizational Change: A Commentary. The Journal of Applied Behavioral Science 42(1), 127-134.

Pirotte, G. \& Poncelet, M. (2002). Éveil des sociétés civiles en milieu urbain et organisations non gouvernementales : les exemples de Cotonou et Lubumbashi. Autrepart 23, 73-88.

Saint-Martin, A. (2010). Luc Boltanski, De la critique. Précis de sociologie de l'émancipation. Paris, Gallimard, 2009, 294 p. Cahiers d'histoire. Revue d'histoire critique, (pp. 112-113). Disponible à : http://journals.openedition.org/chrhc/2193

Thornton, P. H. \& Ocasio, W. (1999). Institutional Logics and the Historical Contingency of Power in Organizations: Executive Succession in the Higher Education Publishing Industry, 1958-1990. American Journal of Sociology 105, 99-122.

Trethewey, G. E. A. \& Ashcraft, K. L. (2004). Special issue introduction: Practicing disorganization: The development of applied perspectives on living with tension. Journal of Applied Communication Research 2(32), 81-88.

Veyne, P. (2008). Foucault Sa pensée, sa personne. Paris : Albin Michel.

Vihalem, M. (2011). Qu'est-ce qu'une subjectivation? Les rapports entre le savoir, le pouvoir et le sujet dans la pensée de Michel Foucault. Synergies. Pays riverains de la Baltique 8, 89-100. Disponible à : https://gerflint.fr/Base/Baltique8/vihalem.pdf

\section{@) $\Theta \Theta \Theta$}

«Attribution - Pas d'Utilisation Commerciale - Pas de Modification 4.0 International» (CC BY-NC-ND) 